\title{
SPERMIOGÉNESE ET FONCTION DU SAC VAGINAL CHEZ ICHTHYOCEPHALUS ANADENOBOLI n. sp. (RHIGONEMATIDAE; NEMATODA), PARASITE D'ANADENOBOLUS POLITUS (PORAT) (RHINOCRICIDAE; DIPLOPODA) EN GUADELOUPE
}

\author{
D. VAN WAEREBEKE*, M. L. ADAMSON** et A. KERMARREC***
}

RÉSUMÉ. Description de Ichthyocephalus anadenoboli n. sp. (Rhigonematidae; Nematoda) trouvé sur Anadenobolus politus (Porat). (Rhinocricidae; Diplopoda) provenant de Guadeloupe. La nouvelle espèce ressemble plus particulièrement à $I$. egleri du fait que les deux sexes sont à peu près de même taille et les spicules très inégaux; elle s'en distingue par le spicule droit beaucoup plus long, par la forme de l'extrêmité du spicule gauche et par l'existence d'un sac vaginal débouchant dans le vagin, à la jonction avec les utérus. Certains aspects de la spermiogénèse de la nouvelle espèce sont étudiés. La maturation des spermatides se déroule suivant deux phases, la première ayant lieu dans le testicule et le réceptacle séminal du mâle, la seconde dans le sac vaginal de la femelle. Au cours de cette seconde phase la forme du spermatide subit de profonds changements en même temps que se transforme son noyau. On peut supposer que le sac vaginal observé chez certaines espèces de Rhigonema et la bourse copulatrice de certains Tetrameres et de Maupasina weissi sont, comme dans le cas de $I$. anadenoboli, des organes spécialisés pour favoriser les derniers stades de la spermiogénèse.

Spermiogenesis and function of the vaginal diverticulum in Ichthyocephalus anadenoboli n. sp. (Rhigonematidae; Nematoda), a parasite of Anadenobolus politus (Porat) (Rhinocricidae; Diplopoda) in Guadeloupe

SUMMARY. Ichthyocephalus anadenoboli n. sp. (Rhigonematidae ; Nematoda) from Anadenobolus politus (Porat) (Rhinocricidae; Diplopoda) from Guadeloupe is described. The new species most closely resembles $I$. egleri since both sexes are of approximately the same size and the spicules are markedly unequal ; it is distinguished in having a much longer right spicule, by the form of the extremity of the left spicule and by the presence of a vaginal sac opening into the vagina at its junction with the uteri. In addition certain aspects of spermiogenesis in the new species are studied. Maturation of spermatids occurs in two phases, one in the testis and seminal receptacle of males, the second in the vaginal sac in females. The second phase involves profound

* Office de Recherche Scientifique et Technique Outre-Mer, Laboratoire de Zoologie (Vers) Muséum National d'Histoire Naturelle, 61, rue Buffon, F 75231 Paris Cedex 05.

** Ecole Pratique des Hautes Études et Laboratoire de Zoologie (Vers), Muséum National d'Histoire Naturelle, 61, rue Buffon, F 75231 Paris Cedex 05.

*** Centre de Recherche des Antilles et de la Guyane, Station de Zoologie et Lutte Biologique, 97170 Petitbourg, Guadeloupe.

Accepté le rer juin 1983 . 
changes in form as well as nuclear transformation. It is suggested that the vaginal sac observed in certain species of Rhigonema as well as the "bourse copulatrice " of certain Tetrameres spp. and Maupasina sveissi, like that of Ichthyocephalus anadenoboli, are specialized to accomodate the final stages of spermiogenesis.

\section{Introduction}

Lors de l'examen de spécimens d'Anadenobolus politus (Porat) de Guadeloupe, de nombreux nématodes appartenant à une nouvelle espèce d'Ichthyocephalus (famille des Rhigonematidae) ont été trouvés. Ichthyocephalus anadenoboli n. sp., se distingue de toutes les autres espèces du genre par la présence chez la femelle d'un sac volumineux qui débouche dans le vagin à sa jonction avec les utérus.

Un tel sac se retrouve chez certaines espèces du genre Rhigonema Cobb, 1898 (Rhigonematidae). Sa fonction est inconnue mais Leidy (1849) le désigne sous le nom de "seminal receptacle " et plus tard (1853), sous le nom de "spermatheca ". Christie et Cobb (1927) remarquent que le véritable réceptacle seminal est situé entre l'ovaire et l'utérus mais signalent que "... elongated cephalated sperms may occur in the unpaired enlargement sometimes present near the vulva ". Nos observations sur $I$. anadenoboli $\mathrm{n}$. sp. montrent que les derniers stades de la spermiogénèse se déroulent dans ce sac vaginal.

\section{Matériels et méthodes}

La description de l'espèce est basée sur des nématodes provenant de l'intestin postérieur de plusieurs spécimens d'Anadenobolus politus (Porat) (Rhinocricidae; Diplopoda) de Guadeloupe. Les nématodes ont été fixés à l'ethanol à $70 \%$ chaud et conservés à l'ethanol à $70 \%$ avant d'être éclaircis et étudiés au lactophénol. Les spécimens types sont conservés dans les collections du Muséum national d'Histoire naturelle de Paris (RA 116).

Des spécimens vivants mâles et femelles d'Ichthyocephalus anadenoboli n. sp. ont été placés individuellement dans une solution de $\mathrm{NaCl}$ à $0,5 \%$ sur des lames ; l'appareil génital a été isolé et le reste du ver éliminé. Un fixateur (6 à $8 \%$ formol dans une solution composée de trois volumes d'éthanol pour un volume d'acide acétique) a été ajouté goutte à goutte sur le spécimen; la lame a été conservée dans du fixateur frais pendant une nuit avant d'être colorée à l'Haematoxyline $(20 \%$ dans une solution à $50 \%$ d'acide proprionique) (voir Adamson, 1981 pour détails).

\section{Ichthyocephalus anadenoboli n. sp.}

Description ( $f g .1$ à 3 )

Généralités : long corps cylindrique. Tête légèrement enflée et aplatie dorsoventralement. Dimorphisme sexuel réduit, portant essentiellement sur la taille plus 
grande de la femelle. Cuticule portant des stries transversales sur tout le corps, excepté la tête et l'extrémité caudale ; bord postérieur de chaque strie portant de petites épines très fines, flexibles, de 2 à $3 \mu \mathrm{m}$ de long vers la tête et dont la taille diminue progressivement vers la queue.

Ouverture buccale étirée latéralement, bordée par une lèvre dorsale et une lèvre ventrale. Amphides situés aux coins de la bouche. 4 papilles céphaliques coniques submédianes.

Cavité buccale limitée par un revêtement cuticulaire armé de nombreuses petites dents formant une rape, comprenant trois lobes dorsaux et deux lobes ventraux ; lobe dorsal médian prolongé par une formation cuticulaire en forme d'une glotte obturant la lumière œesophagienne. Lumière œsophagienne d'abord aplatie en arrière de la glotte puis devenant triradiée dans la partie postérieure du corpus. Neuf cellules en arcades situées autour de la partie postérieure du corpus.

Mâle : mensurations (premier chiffre concernant l'holotype, chiffres entre parenthèses concernant les extrêmes et la moyenne des dix spécimens étudiés). Longueur $8,23(6,94-8,97 ; 8,09) \mu \mathrm{m}$. Largeur maximum $230(188-287 ; 334) \mu \mathrm{m}$. Distance de l'extrémité antérieure du corps à la jonction œsophago-intestinale 467 (418$476 ; 445) \mu \mathrm{m}$. Corpus $207 \mu \mathrm{m}$ de long et $141 \mu \mathrm{m}$ de large. Isthme $36 \mu \mathrm{m}$ de long et $68 \mu \mathrm{m}$ de large. Bulbe $107 \mu \mathrm{m}$ de long et $111 \mu \mathrm{m}$ de large. Queue 940 (560-1 020; 760) $\mu \mathrm{m}$, spicule droit $737(705-842 ; 770) \mu \mathrm{m}$, spicule gauche $180(160-193 ; 178) \mu \mathrm{m}$. Épaisseur spicule gauche $39(33-41 ; 37) \mu \mathrm{m}$. Pore excréteur à $332 \mu \mathrm{m}$ et anneau nerveux à $357 \mu \mathrm{m}$ de l'extrémité antérieure.

Stries de la cuticule distantes de 3 à $5 \mu \mathrm{m}$; épines s'estompant progressivement dans la région ventrale avant et après l'anus. Testicule replié sur lui-même au niveau du premier tiers de la longueur du corps, prolongé par la vésicule séminale contenant de nombreux spermatides de grande taille, elle-même séparée par un étranglement d'une zone glandulaire occupant près des deux cinquièmes de la longueur du corps. Spicules très inégaux, le spicule droit long et mince étant soutenu par le spicule gauche court et épais jouant le rôle d'un gubernaculum en guidant le spicule qu'il enserre par sa pointe et ses deux lobes latéraux.

24 à 29 papilles réparties de la manière suivante :

- 6 (chez 6 spécimens) à 7 (4 spécimens) préanales droites ;

- 7 (7 spécimens) à 10 (2 spécimens avec $8 ; 1$ spécimen avec 10$)$ préanales gauches ;

- 6 postanales droites ;

- 5 (8 spécimens) à 6 ( 2 spécimens) postanales gauches.

(soit 3 spécimens avec 24 papilles, 6 spécimens avec 25 papilles et un spécimen avec 29 papilles).

Femelle : mensurations (allotype suivi par les extrêmes et la moyenne de neuf paratypes entre parenthèses). Longueur $10,1(9,0-11,0 ; 10,5) \mathrm{mm}$. Largeur maximum $306(280-390 ; 328) \mu \mathrm{m}$. Distance de l'extrémité antérieure du corps à la jonction œsophago-intestinale $476(447-526$; 497) $\mu \mathrm{m}$. Corpus $259 \mu \mathrm{m}$ de long et $159 \mu \mathrm{m}$ de large en vue latérale. Isthme $40 \mu \mathrm{m}$ de long et $84 \mu \mathrm{m}$ de large. Bulbe $111 \mu \mathrm{m}$ de long et $127 \mu \mathrm{m}$ de large. Queue $980(870-1320 ; 1100) \mu \mathrm{m}$ de long. Pore excréteur à $314 \mu \mathrm{m}$, 


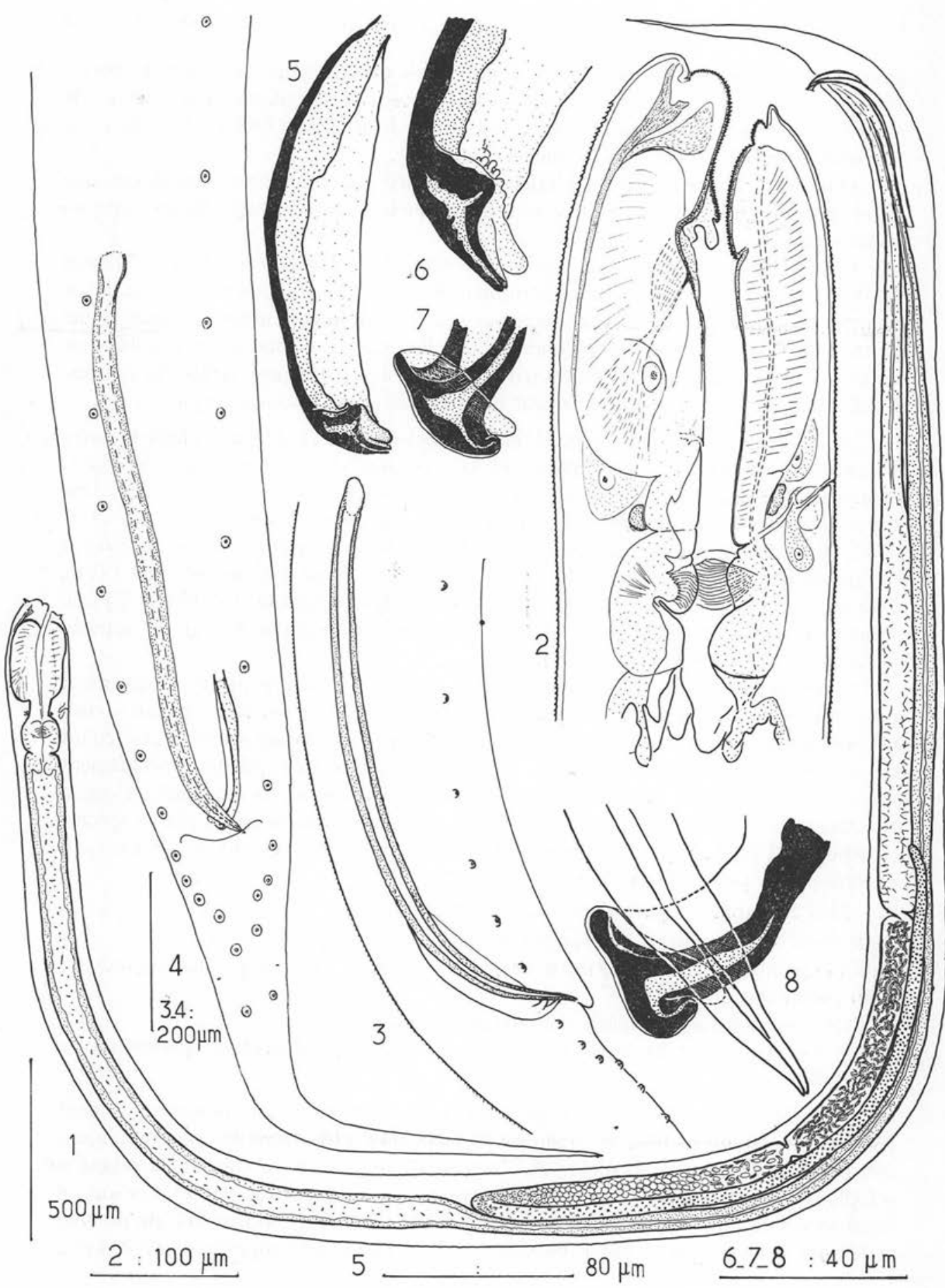

FIG. I. - Ichthyocephalus anadenoboli n. sp. Mâle. I, vue latérale. 2, œsophage, coupe sagittale. 3 , région postérieure, vue latérale droite. 4 , région postérieure, vue ventrale. 5 , spicule gauche, vue latérale. 6 , spicule gauche, vue latérale, détail de l'extrémité distale. 7 , spicule gauche, vue ventrale, détail de l'extrémité distale. 8 , extrémité des deux spicules, vue ventrale. 

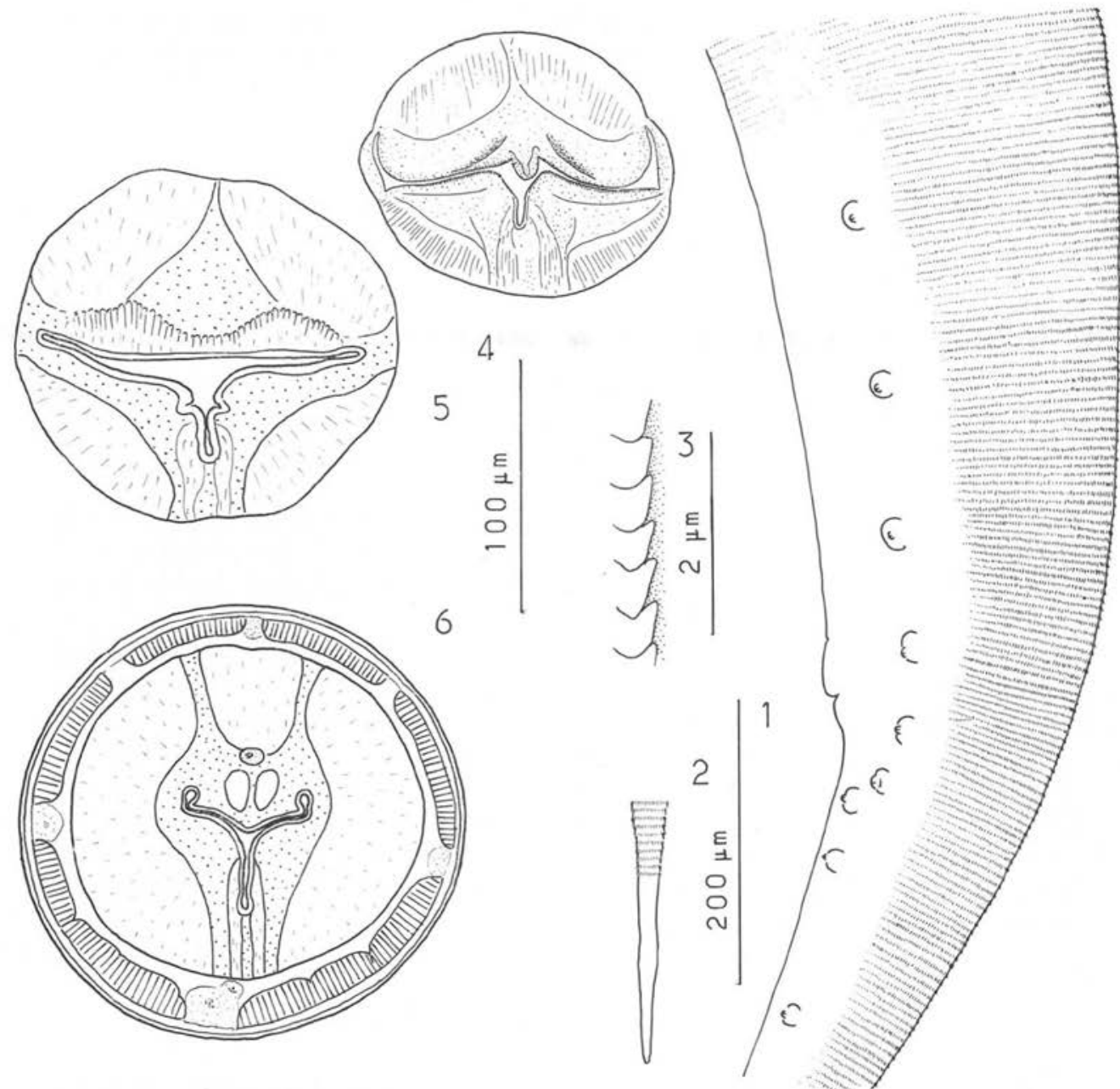

FIG. 2. - Ichthyodephalus anadenoboli n. sp. Mâle. I, région postérieure, vue latérale gauche. 2, extrémité caudale. 3, épines, vue latérale (région œsophagienne). 4, coupe transversale du corpus au niveau de la " glotte ». 5, coupe transversale du corpus en arrière de la glotte. 6 , coupe transversale du corps au niveau de la partie postérieure du corpus.

anneau nerveux à $354 \mu \mathrm{m}$ et vulve à $5,3(5,0-6,0 ; 5,4) \mathrm{mm}$ de l'extrémité antérieure. Eufs 133-139 $\mu \mathrm{m}$ de long et 83-86 $\mu \mathrm{m}$ de large (dix œufs mesurés).

Épines également réparties sur toute la surface du corps; stries de la cuticule distantes de 4,5-7,0 $\mu \mathrm{m}$; deux ovaires prenant leur origine en avant de la vulve, repliés une fois sur eux-mêmes avant de déboucher dans les deux réceptacles séminaux contenant un petit nombre de spermatozoïdes. Deux utérus remplis d'œufs (de 30 
à plus de cent par femelle) débouchant dans le vagin ; vagin prolongé en arrière de la jonction avec les utérus par une poche volumineuse (sac vaginal) renfermant des spermatides. Vulve non saillante.

\section{Discussion}

Le genre Ichthyocephalus Artigas, 1926 renferme cinq espèces : l'espèce type du genre, I. ichthyocephalus Artigas, 1926, I. ichthyocephaloides Dollfus, 1952, (espèce que nous considérons comme species inquirenda, le mâle étant inconnu et la femelle insuffisament décrite $\left.{ }^{1}\right)$, I. egleri Travassos \& Kloss, 1958 I. antenori Travassos et Kloss, 1959 et $I$. seymouri Adamson, 1983.

Ichthyocephalus anadenoboli $\mathrm{n}$. sp. est proche de $I$. egleri. Ce sont les deux seules espèces du genre chez lesquelles les spicules sont très inégaux et dont le mâle est à peine plus petit que la femelle (alors que la différence de taille chez les autres espèces excepté $I$. antenori, est dans le rapport de 1 à 3 ).

La nouvelle espèce se distingue facilement de $I$. egleri puisque la différence de taille entre les spicules est beaucoup plus marquée ( 1 à $4 \mathrm{chez} I$. anadenoboli et 1 à 2 chez $I$. egleri). De plus, la nouvelle espèce est beaucoup plus grande que les autres espèces du genre et est la seule à posséder un sac vaginal.

Le sac vaginal de $I$. anadenoboli est certainement homologue à celui trouvé chez certaines espèces de Rhigonema ; cependant, il en diffère légèrement car, chez Rhigonema les deux utérus se réunissent en une trompe débouchant à la base du sac vaginal; chez $I$. anadenoboli ils débouchent séparément à la base du sac vaginal. Ce sac ressemble beaucoup à ce que Seurat (1920) appelle " bourse copulatrice " et que l'on trouve chez certaines espèces de Tetrameres (Tetrameridae; Spirurida) et chez Maupasina weissi (Maupasinidae; Ascaridida), mais nous préférons éviter l'utilisation de cette expression utilisée en entomologie pour désigner une structure jouant le rôle du réceptacle séminal.

\section{Spermiogénèse chez I. anadenoboli}

La maturation des spermatides comprend deux phases; la première intervient dans l'extrémité postérieure du testicule et dans la vésicule séminale du mâle; la seconde intervient dans le sac vaginal de la femelle.

\section{Phase I}

Les spermatides jeunes sont à peu près sphériques; le matériel nucléaire se présente sous forme d'une masse excentrée, très aplatie, aux contours irréguliers, dont la coloration à l'haematoxyline est homogène (fig. $4 \mathrm{~A}$ ). Les spermatides avant

r. Les spécimens types de cette espèce sont conservés en montage permanent dans les collections du Muséum national d'Histoire naturelle de Paris (Laboratoire de zoologie-Vers : BD 8). Ils sont fortement éclaircis et bien que l'anatomie soit difficile à observer nous avons pu constater qu'il n'y a pas de sac vaginal. 


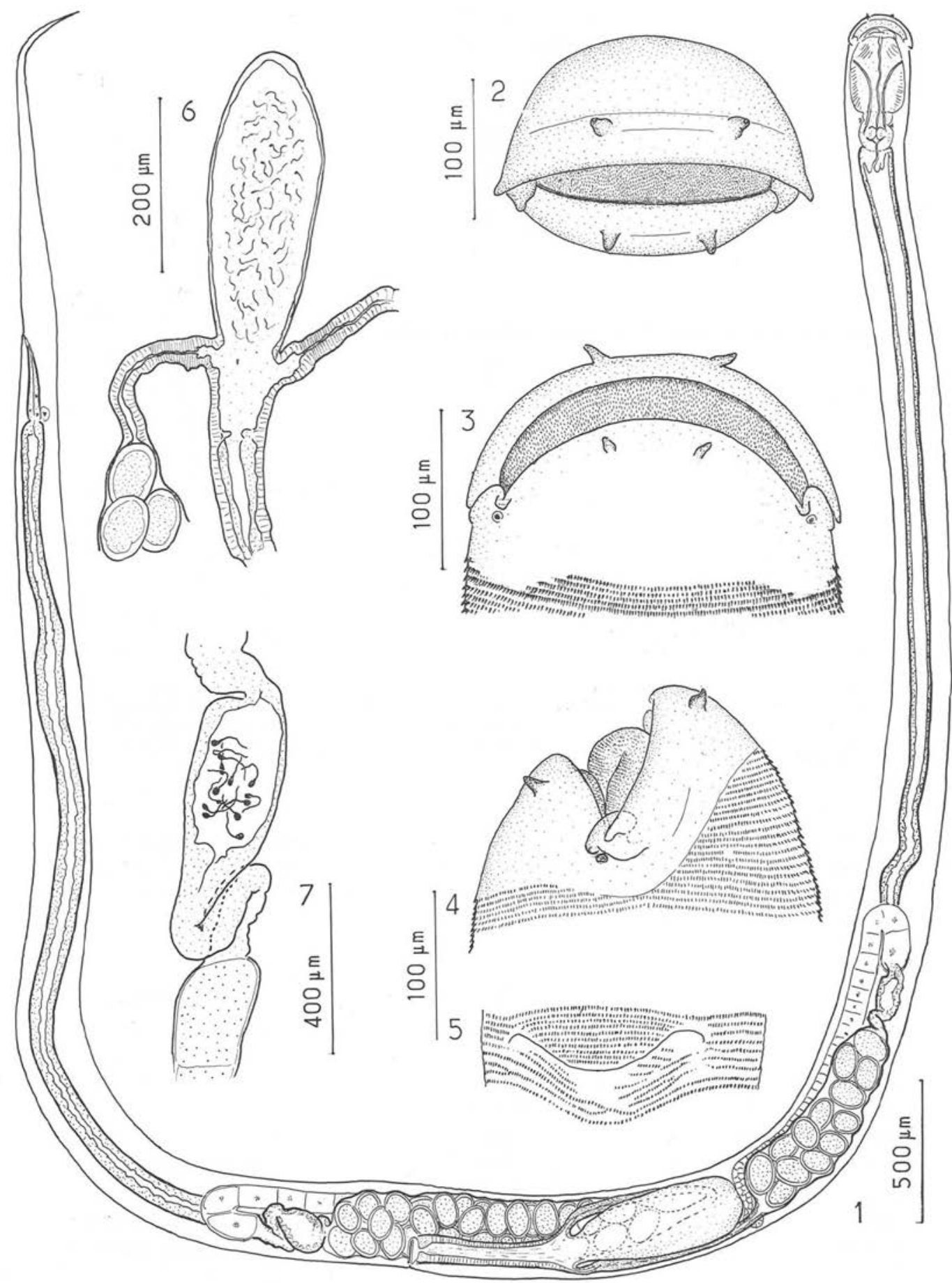

FIG. 3. - Ichthyocephalus anadenoboli n. sp. Femelle. I, vue latérale. 2, tête, vue apicale 3, tête, vue ventrale. 4 , tête, vue latérale. 5 , région anale, vue ventrale. 6 , sac vaginal 7 , réceptacle séminal. 


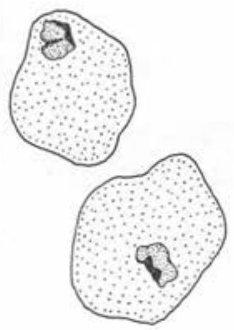

A



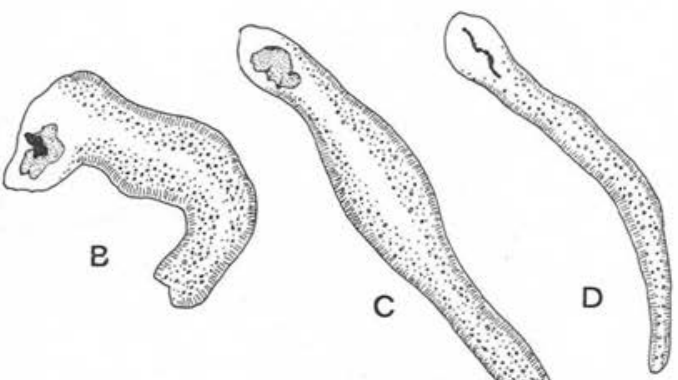



FIG. 4. - Ichthyocephalus anadenoboli n. sp., spermiogénèse. A à F, matériel coloré à l'hématoxyline et écrasé. A à D, évolution chez le mâle. E, différents stades de l'évolution nucléaire des spermatides dans le sac vaginal (ordre hypothétique de a à h). F, spermatozoïde mûr dans le réceptacle séminal. G à J, matériel disséqué dans le lactophénol ; évolution des spermatides dans le sac vaginal.

d'entrer dans la vésicule séminale, s'allongent; de nombreuses formations apparaissent à la périphérie du cytoplasme, lui donnant un aspect strié ( $f i g .4 B$ à $D$ ). Les spermatides dans la vésicule séminale mesurent 60 à $70 \mu \mathrm{m}$ de long et jusqu'à $10 \mu \mathrm{m}$ de large.

\section{Phase II}

Le sac vaginal contient des spermatides à différents stades d'évolution. Les stades les plus précoces ressemblent à ceux vus dans la vésicule séminale du mâle et le développement ultérieur consiste en un allongement progressif de la "queue » du spermatide ( fig. $4 \mathrm{H} \grave{a} J$ ). En même temps le noyau subit de profonde transformations (fig. $4 E$ à $H$ ) ; d'abord aplati et irrégulier il devient progressivement plus allongé et plus coloré. Puis il se condense pour former dans l'extrémité " céphalique " du spermatide une masse très colorée et discoïdale. Les spermatozoïdes mûrs ( $f i g .4 F \grave{a} J$ ) ont été observé dans le réceptacle séminal aussi bien que dans le sac vaginal. Ils possèdent une petite " tête " ronde capable d'émettre des pseudopodes et contenant une masse de chromatine en forme de disque, et une longue queue filiforme. 


\section{Conclusions}

Dès 1906 Stuckmann étudiant Strongylus filaria notait que les spermatozoïdes dans le vagin étaient différents de ceux vus dans le réceptacle séminal de la femelle. Des études récentes (Foor 1970; 1971; McLaren, 1973; Durette-Desset, 1973) montrent que chez plusieurs groupes de nématodes les spermatides subissent des transformations dans l'appareil génital de la femelle. Foor (1970) souligne les changements intervenant chez les spermatides d'un certain nombre de nématodes après l'insémination : fusion de gouttelettes lipidiques, libération du contenu de petits organites membraneux périphériques, changement de la forme et acquisition de la mobilité.

La spermiogénèse chez $I$. anadenoboli comprend deux phases dont une se passe chez le mâle et la deuxième dans le sac vaginal de la femelle. Cette deuxième phase comprend non seulement des changements de la forme mais aussi des transformations nucléaires et est probablement stimulée par des sécrétions du sac vaginal. Vraisemblablement le sac vaginal chez certaines espèces de Rhigonema aussi bien que la bourse copulatrice chez Tetrameres fissispina et Maupasina weissi sont spécialisés pour permettre les derniers stades de la spermiogénèse.

\section{BIBLIOGRAPHIE}

Adamson M. L. : Studies on gametogenesis in Gyrinicola batrachiensis (Walton, 1929) (Oxyuroidea; Nematoda). Can. J. Zool., 1981, s9, 1368-1376.

Christie J R., СовB N. A. : Rhigonema (Isacis Scrjabin, 1914; Isacis Baylis and Daubney, I926 ; nec Isacis Lespes, 1856 ) nemas inhabiting the intestine of millipedes. J. Wash. Acad. Sci., $1927,17,17-19$.

DurEtTE-DESSET M. C. : Redescription d'Heligmosomoides travassosi Schulz, I9r6 (Nématode Héligmosominé) parasite de Cricetus cricetus L. (Cricetidae). Ann. Parasitol. Hum. Comp., $1973,48,483-488$.

Foor W. E. : Spermatozoan Morphology and zygote formation in nematodes. Biol. Reprod. Suppl., I970, 2, I77-202.

Foor W. E., Johnson M. H., BEAver P. C. : Morphological changes in the spermatozoa of Dipetalonema viteae in utero. J. Parasitol., I971, 57, II63-1 I69.

LeIDY J. : On the existence of Entophyta in healthy animals as a natural condition. Proc. Acad. Nat. Sci. Philadelphia, 1849, 4, 225-233.

Leidy J. : A flora and fauna within living animals. Smithsonian Contributions to Knowledge, 1853 , S, $1-67$.

MCLAREN D. L. : The structure and development of the spermatozoon of Dipetalonema viteae (Nematoda; Filarioidea). Parasitology, 1973, 66, 447-463.

Seurat L. G. : Histoire naturelle des nématodes de la Berbérie. I re partie. Morphologie, développement, éthologie et affinités des nématodes. Université d'Alger. Faculté des Sciences. Fondation J. Azoubib. Travaux du laboratoire de zoologie générale, 1920, $22 \mathrm{I}+\mathrm{VI}$ p. 